\title{
The temporal behaviour of the hydrodynamic force on a body in response to an abrupt change in velocity at small but finite Reynolds number
}

\author{
By PHILLIP M. LOVALENTI† AND JOHN F. BRADY \\ Division of Chemistry and Chemical Engineering, California Institute of Technology, Pasadena, \\ CA 91125, USA
}

(Received 29 March 1994 and in revised form 8 February 1995)

A modification to the $O(R e)$-accurate expression for the hydrodynamic force acting on a body in arbitrary time-dependent motion, determined by Lovalenti \& Brady (1993), is presented. This simple modification captures the $O\left(R e^{2}\right)$ transient behaviour of the force, which has been recently shown to dominate at large time (Lawrence \& Mei 1995), while maintaining the overall $O(R e)$ accuracy.

\section{Introduction}

In a recent paper by Lovalenti \& Brady (1993) (henceforth referred to as LB), the authors derive an $O(R e)$-accurate expression for the hydrodynamic force on bodies in arbitrary motion in an unbounded time-dependent uniform flow. Here the Reynolds number, $R e$, is based on the characteristic body dimension and the velocity of the body relative to the local fluid velocity. The expression exhibits both algebraic and exponential decay in response to step changes in the speed of the body. The algebraic decay is observed for the case of a body accelerating from rest, a $t^{-2}$ decay, and for a body coming to an abrupt stop, a $t^{-1}$ decay, both of which are faster than the $t^{-1 / 2}$ decay predicted by the unsteady Stokes equations which neglect convective inertial effects. For the intermediate cases between these two extremes an exponential decay is obtained.

In a more recent paper, Lawrence \& Mei (1995) (henceforth referred to as LM) showed keen insight in considering the long-time decay of the drag on a body as steady state is approached for more general magnitudes of the Reynolds number. In all cases they find that the decay is algebraic, either $t^{-1}$ or $t^{-2}$. The $t^{-1}$ behaviour is obtained for the stopping problem mentioned above as well as for the case of a body making an exact reversal in its direction of motion. For all other cases the decay is $t^{-2}$. For the conditions found by LB to exhibit exponential decay, LM find an $O\left(R e^{2}\right)$ contribution that decays as $t^{-2}$ for small Reynolds number. This result implies that the $O\left(R e^{2}\right)$ contribution to the transient part of the drag will ultimately dominate the $O(R e)$ contribution under these conditions because the $O(R e)$ contribution decays exponentially fast. This $O\left(R e^{2}\right)$ contribution will dominate when $t^{\prime} U^{2} / v \gg \ln (1 / R e)$, where $t^{\prime}$ is dimensional time, $v$ is the kinematic viscosity of the fluid and $U$ is the characteristic velocity of the body. At that point, for small Reynolds number, the transient part of the drag will be very small - smaller than $O\left(R e^{2}\right) / \ln (R e)^{2}$ relative 
to the Stokes drag. As the Reynolds number approaches $O(1)$, however, it becomes more important to take these higher-order transient effects into account. In this paper we present a very simple extension of the LB theory which correctly captures the $O\left(R e^{2}\right) t^{-2}$ transient behaviour.

\section{The modified unsteady Oseen force}

The hydrodynamic force $F(t)$, accurate to $O(R e)$, acting on a spherical particle undergoing arbitrary motion in a uniform flow found by LB is

$$
\boldsymbol{F}(t)=\boldsymbol{F}_{S t}(t)+\boldsymbol{F}_{A M}(t)+m_{f} \dot{U}^{\infty}(t)+\boldsymbol{F}_{U O S}(t)+o(R e),
$$

where $\boldsymbol{F}_{S t}(t)$ and $\boldsymbol{F}_{A M}(t)$ represent the familiar Stokes drag and added mass, respectively. Here $m_{f}$ is the mass of the fluid displaced by the particle, and $\dot{U}^{\infty}(t)$ is the acceleration of the uniform flow. We refer to $\boldsymbol{F}_{U O_{S}}(t)$ as the unsteady Oseen force since it reduces to the steady Oseen correction to the Stokes drag for steady motion. For finite values of the Reynolds number, $\boldsymbol{F}_{U o_{S}}(t)$ replaces the history integral from the solution to the unsteady Stokes equations known as the Basset force. Although it behaves as the Basset force for short-time-scale motion, $\boldsymbol{F}_{U O_{S}}(t)$ behaves very differently for longer time scales owing to the convective transport mechanisms neglected in the unsteady Stokes equations.

Except for the addition of a lift force term for nonspherical bodies (which is proportional to the square of the slip velocity), an expression analogous to (2.1) exists for bodies of arbitrary shape. The reader is referred to the original LB paper for the details. An important aspect of the analysis is that the form of the unsteady Oseen force is identical for all bodies and is given by

$$
\begin{aligned}
\boldsymbol{F}_{U O S}(t)= & \frac{3}{8} \operatorname{Re} \Phi \cdot\left\{\int _ { - \infty } ^ { t } \left[\frac{2}{3} \boldsymbol{F}_{S t}^{\dagger}(t)-\left\{\frac{1}{|\boldsymbol{A}|^{2}}\left(\frac{\pi^{1 / 2}}{2|\boldsymbol{A}|} \operatorname{erf}(|\boldsymbol{A}|)-\exp \left(-|\boldsymbol{A}|^{2}\right)\right)\right\} \boldsymbol{F}_{S t}^{\|}(s)\right.\right. \\
& \left.+\frac{2}{3} \boldsymbol{F}_{S t}^{\perp}(t)-\left\{\exp \left(-|\boldsymbol{A}|^{2}\right)-\frac{1}{2|\boldsymbol{A}|^{2}}\left(\frac{\pi^{1 / 2}}{2|\boldsymbol{A}|} \operatorname{erf}(|\boldsymbol{A}|)-\exp \left(-|\boldsymbol{A}|^{2}\right)\right)\right\} \boldsymbol{F}_{S t}^{\perp}(s)\right] \\
& \left.\times \frac{2 \mathrm{~d} s}{\pi^{1 / 2}(t-s)^{3 / 2}}\right\} .
\end{aligned}
$$

Time $t$ and the dummy variable $s$ have been nondimensionalized by $v / U^{2}$, and $A$ is a vector that is related to the relative displacement of the body from time $s$ to $t$ :

$$
A(t, s)=\frac{1}{2}(t-s)^{-1 / 2} \int_{s}^{t} U_{s}(q) \mathrm{d} q
$$

where the slip velocity $\boldsymbol{U}_{s}(t)$ is the velocity of the body relative to the local fluid velocity at time $t$ and has been nondimensionalized by the characteristic speed $U$. Also $\Phi$ represents the Stokes resistance tensor nondimensionalized by $6 \pi \mu a$ where $\mu$ is the viscosity of the fluid and $a$ is the characteristic body dimension (the radius in the case of a sphere). Thus, for a sphere, $\Phi$ is the idem tensor. The vector quantities $\boldsymbol{F}_{S t}^{\|}(s)$ and $\boldsymbol{F}_{S t}^{\perp}(s)$ are the components of the pseudo-steady Stokes drag respectively parallel and perpendicular to the displacement vector $A(t, s)$. For the remainder of the paper all forces will be nondimensionalized by $6 \pi \mu a U$; therefore, in the case of a sphere, $F_{S t}(t)$ is given simply by the negative of the nondimensional slip velocity. The Reynolds number is defined by $R e=a U / v$. 


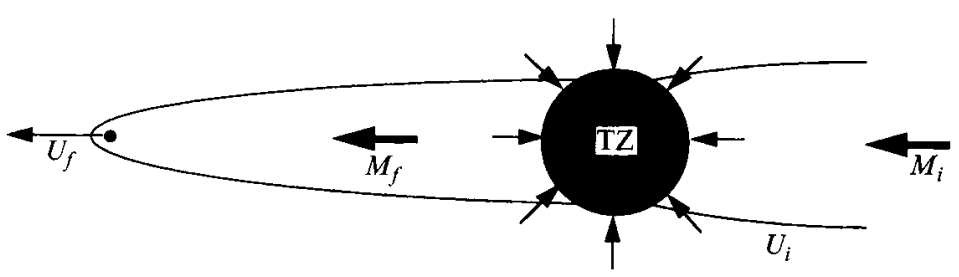

FIgURE 1. Schematic diagram of the long-time wake structure after a step increase in the speed of a body from $U_{i}$ to $U_{f}$. 'TZ' labels the transition zone where the speed change occurred and represents a sink flow to make up for the difference in the mass fluxes $\left(M_{i}\right.$ and $\left.M_{f}\right)$ in the two adjoining wakes. While the mass fluxes in the wakes are both $O(\mu a)$ to leading order, their difference is $O\left(\mu a\left(R e_{f}-R e_{i}\right)\right)$, provided the initial velocity is nonzero. This difference is also the magnitude of the sink flow which is responsible for the $t^{-2}$ decay as the body moves farther away from the transition zone. The figure has been adapted from LM, and see that article for a more detailed discussion of the phenomena.

Because we will be focusing on the force response to impulsive changes in the velocity of the body (or fluid) between two steady values, terms such as the added mass, which are proportional to the acceleration of the body (or fluid), will appear as Dirac delta functions at the time of the velocity change. Therefore, for the purposes of this paper, all the important transient effects will come from the unsteady Oseen force.

Now we define a history force $\boldsymbol{F}_{h}(t)$ which decays to zero when approaching steady state by subtracting from (2.2) the steady Oseen correction to obtain

$$
\begin{aligned}
\boldsymbol{F}_{h}(t)= & \frac{3}{8} \operatorname{Re} \Phi \cdot\left\{\int _ { - \infty } ^ { t } \left[\frac{2}{3} \boldsymbol{F}_{S t}^{\|}(t)-\left\{\frac{1}{|\boldsymbol{A}|^{2}}\left(\frac{\pi^{1 / 2}}{2|\boldsymbol{A}|} \operatorname{erf}(|\boldsymbol{A}|)-\exp \left(-|\boldsymbol{A}|^{2}\right)\right)\right\} \boldsymbol{F}_{S t}^{\|}(s)\right.\right. \\
& \left.+\frac{2}{3} \boldsymbol{F}_{S t}^{\perp}(t)-\left\{\exp \left(-|\boldsymbol{A}|^{2}\right)-\frac{1}{2|\boldsymbol{A}|^{2}}\left(\frac{\pi^{1 / 2}}{2|\boldsymbol{A}|} \operatorname{erf}(|\boldsymbol{A}|)-\exp \left(-|\boldsymbol{A}|^{2}\right)\right)\right\} \boldsymbol{F}_{S t}^{\perp}(s)\right] \\
& \left.\times \frac{2 \mathrm{~d} s}{\pi^{1 / 2}(t-s)^{3 / 2}}-\left(\boldsymbol{F}_{S t}^{\|}(t)+\frac{3}{2} \boldsymbol{F}_{S t}^{\perp}(t)\right)\left|\boldsymbol{U}_{s}(t)\right|\right\}
\end{aligned}
$$

It is this expression that exhibits exponential decay for step changes in velocity, except for the starting, stopping, or reversal of motion problems previously discussed which have algebraic decay. With regard to long-time transient effects, the above history integral can be roughly divided into two parts for a step change in velocity at time zero: the part from $s=-\infty$ to $s=0$ represents effects from the old wake generated by the motion of the body for $s<0$, while that from $s=0$ to $s=t$ represents the contributions to the drag from the new wake created by the body motion for $s>0$. For long times these two parts can nearly negate each other and lead to exponential decay. This near balance of contributions occurs because the mass fluxes in the old and new wakes are identical to lowest order in Reynolds number, being $O(F / U) \sim O(\mu a)$. At higher-order they are not equal, and this difference is what accounts for the $O\left(R e^{2}\right)$ contribution discussed above. (See the schematic diagram in figure 1.)

The reason the Stokes quantities $\boldsymbol{F}_{S t}^{\|}$and $\boldsymbol{F}_{S t}^{\perp}$ arise in (2.4) is that the equation is derived from the point-forced Navier-Stokes equations where the magnitude of the point force was chosen as the Stokes drag. Since we wish to capture higher-order effects, a reasonable solution would be to replace these Stokes quantities with their 
steady Oseen-corrected counterparts, thereby producing a higher-order-accurate representation of the point force, which describes the dominant hydrodynamic influence of the body on the fluid. Thus we define a modified history force by the following expression:

$$
\begin{aligned}
\boldsymbol{F}_{h}^{\prime}(t)= & \frac{3}{8} \operatorname{Re} \Phi \cdot\left\{\int _ { - \infty } ^ { t } \left[\frac{2}{3} \boldsymbol{F}_{O s}^{\|}(t)-\left\{\frac{1}{|\boldsymbol{A}|^{2}}\left(\frac{\pi^{1 / 2}}{2|\boldsymbol{A}|} \operatorname{erf}(|\boldsymbol{A}|)-\exp \left(-|\boldsymbol{A}|^{2}\right)\right)\right\} \boldsymbol{F}_{O s}^{\|}(s)\right.\right. \\
& \left.+\frac{2}{3} \boldsymbol{F}_{O s}^{\perp}(t)-\left\{\exp \left(-|\boldsymbol{A}|^{2}\right)-\frac{1}{2|\boldsymbol{A}|^{2}}\left(\frac{\pi^{1 / 2}}{2|\boldsymbol{A}|} \operatorname{erf}(|\boldsymbol{A}|)-\exp \left(-|\boldsymbol{A}|^{2}\right)\right)\right\} \boldsymbol{F}_{O s}^{\perp}(s)\right] \\
& \left.\times \frac{2 \mathrm{~d} s}{\pi^{1 / 2}(t-s)^{3 / 2}}-\left(\boldsymbol{F}_{O s}^{\|}(t)+\frac{3}{2} \boldsymbol{F}_{O s}^{\perp}(t)\right)\left|\boldsymbol{U}_{s}(t)\right|\right\}
\end{aligned}
$$

where $\boldsymbol{F}_{O s}^{\|}(s)$ and $\boldsymbol{F}_{O s}^{\perp}(s)$ are the components of the pseudo-steady Oseen force:

$$
\boldsymbol{F}_{O s}(t)=\boldsymbol{F}_{S t}(t)+\frac{3}{8} \operatorname{Re}\left|\boldsymbol{U}_{s}(t)\right| \boldsymbol{\Phi} \cdot\left(\boldsymbol{F}_{S t}^{\|}(t)+\frac{3}{2} \boldsymbol{F}_{S t}^{\perp}(t)\right),
$$

respectively parallel and perpendicular to the displacement vector $\boldsymbol{A}(t, s)$. In this way the contributions from the old and new wakes will not balance identically because of the introduction of the nonlinear (in velocity) effect of the Oseen correction. The corresponding modified unsteady Oseen force is now

$$
\begin{aligned}
\boldsymbol{F}_{U O S}^{\prime}(t)= & \frac{3}{8} \operatorname{Re} \Phi \cdot\left\{\int _ { - \infty } ^ { t } \left[\frac{2}{3} \boldsymbol{F}_{O S}^{\|}(t)-\left\{\frac{1}{|\boldsymbol{A}|^{2}}\left(\frac{\pi^{1 / 2}}{2|\boldsymbol{A}|} \operatorname{erf}(|\boldsymbol{A}|)-\exp \left(-|\boldsymbol{A}|^{2}\right)\right)\right\} \boldsymbol{F}_{O S}^{\|}(s)\right.\right. \\
& \left.+\frac{2}{3} \boldsymbol{F}_{O S}^{\perp}(t)-\left\{\exp \left(-|\boldsymbol{A}|^{2}\right)-\frac{1}{2|\boldsymbol{A}|^{2}}\left(\frac{\pi^{1 / 2}}{2|\boldsymbol{A}|} \operatorname{erf}(|\boldsymbol{A}|)-\exp \left(-|\boldsymbol{A}|^{2}\right)\right)\right\} \boldsymbol{F}_{O S}^{\perp}(s)\right] \\
& \left.\times \frac{2 \mathrm{~d} s}{\pi^{1 / 2}(t-s)^{3 / 2}}\right\}
\end{aligned}
$$

The results for the history force for the two different 'point forcings' are shown in tables $1(a)$ and $1(b)$ for two different step changes in the speed of a sphere. The history integrals were evaluated using Mathematica. For comparison, the results from the finite difference solution of $\mathrm{LM}$ are also presented. The quantity $K(t)$ is defined by

$$
K(t)=\left|\boldsymbol{F}_{h}(t)\right| /(1-b),
$$

where $b$ is the ratio of the initial speed to the final speed. Note that the characteristic velocity $U$ is chosen as the final speed. The Oseen point-forced results compare favourably with the LM numerical data over the entire time range, with better agreement for the lower Reynolds number case in table 1. The Stokes point-forced solution, on the other hand, deviates appreciably from the other results, by orders of magnitude, for large times. These data are also plotted in figures 2 and 3 on both linear and log scales. Figure 2(a) shows the very good agreement between the Stokes point-forced solution (2.4) (from the unmodified unsteady Oseen force) and the other two solutions over a wide time range, which demonstrates the fact that at low Reynolds number the unmodified unsteady Oseen force performs well except for large times when the history force contribution to the total hydrodynamic force has already become vanishingly small. This finding does not hold true when the Reynolds number approaches $O(1)$ or larger, as figure $3(a)$ reveals larger deviations between 


\begin{tabular}{|c|c|c|c|c|}
\hline & \multirow[t]{2}{*}{$t^{\prime} U / a$} & \multicolumn{3}{|c|}{ History force: $K(t)$} \\
\hline & & Stokes pt-force & Oseen pt-force & Lawrence \& Mei \\
\hline \multirow{16}{*}{ (a) } & 0.100 & 0.619 & 0.666 & 0.85 \\
\hline & 0.178 & 0.447 & 0.481 & 0.53 \\
\hline & 0.316 & 0.318 & 0.343 & 0.34 \\
\hline & 0.562 & 0.223 & 0.240 & 0.25 \\
\hline & 1.00 & 0.152 & 0.164 & 0.17 \\
\hline & 1.78 & 0.100 & 0.108 & 0.12 \\
\hline & 3.16 & 0.0625 & 0.0679 & 0.07 \\
\hline & 5.62 & 0.0363 & 0.0396 & 0.04 \\
\hline & 10.0 & 0.0189 & 0.0208 & 0.02 \\
\hline & 17.8 & 0.00828 & 0.00927 & 0.01 \\
\hline & 31.6 & 0.00278 & 0.00323 & 0.0037 \\
\hline & 56.2 & 0.000608 & 0.000778 & 0.0009 \\
\hline & 100 & $6.49 \times 10^{-5}$ & 0.000121 & 0.00014 \\
\hline & 178 & $2.04 \times 10^{-6}$ & $1.98 \times 10^{-5}$ & $2.1 \times 10^{-5}$ \\
\hline & 300 & $1.46 \times 10^{-8}$ & $6.26 \times 10^{-6}$ & $6.5 \times 10^{-6}$ \\
\hline & 1000 & $1.12 \times 10^{-19}$ & $5.625 \times 10^{-7}$ & $5.6 \times 10^{-7} *$ \\
\hline \multirow[t]{16}{*}{ (b) } & 0.100 & 0.958 & 1.30 & \\
\hline & 0.178 & 0.653 & 0.896 & \\
\hline & 0.316 & 0.430 & 0.596 & 0.5 \\
\hline & 0.562 & 0.270 & 0.379 & 0.38 \\
\hline & 1.00 & 0.158 & 0.227 & 0.21 \\
\hline & 1.78 & 0.0843 & 0.125 & 0.13 \\
\hline & 3.16 & 0.0388 & 0.0611 & 0.06 \\
\hline & 5.62 & 0.0144 & 0.0252 & 0.027 \\
\hline & 10.0 & 0.00383 & 0.00843 & 0.0085 \\
\hline & 17.8 & 0.000598 & 0.00229 & 0.0027 \\
\hline & 31.6 & $3.93 \times 10^{-5}$ & 0.000599 & 0.00061 \\
\hline & 56.2 & $6.05 \times 10^{-7}$ & 0.000178 & 0.00018 \\
\hline & 100 & $7.71 \times 10^{-10}$ & $5.63 \times 10^{-5}$ & $5.0 \times 10^{-5}$ \\
\hline & 178 & $1.26 \times 10^{-14}$ & $1.78 \times 10^{-5}$ & $1.6 \times 10^{-5}$ \\
\hline & 300 & $9.0 \times 10^{-22}$ & $6.25 \times 10^{-6}$ & $5.0 \times 10^{-6}$ \\
\hline & 1000 & $5.21 \times 10^{-61 *}$ & $5.61 \times 10^{-7}$ & $4.0 \times 10^{-7} *$ \\
\hline
\end{tabular}

TABLE 1. Comparison of the history force obtained from the present solution (2.5) with that from the previous result of Lovalenti \& Brady (2.4) and the numerical results of Lawrence \& Mei for a sphere making a step change in its speed at $t^{\prime}=0$ from $R e_{d}=0.1$ to $R e_{d}=0.3$ in $(a)$, and $R e_{d}=0.8$ to $R e_{d}=1.0$ in $(b)$, where $R e_{d}$ is the Reynolds number based on sphere diameter. Here $t^{\prime}$ indicates dimensional time and an asterisk (*) denotes a solution obtained from the long-time asymptote.

the Stokes point-forced solution and the other two. Figures $2(b)$ and $3(b)$ show the improved agreement with the LM data by the use of the Oseen point-forced solution (2.5) (employing the modification of the unsteady Oseen force) particularly for large time. It should be pointed out that the LM data were not expected by Lawrence $\&$ Mei to be accurate (because of numerical approximations) at small times or at large dimensionless time $\left(t^{\prime} U / a\right)$ of greater than about 300 , which may be partly responsible for the deviations between the solutions.

Although the agreement of the Oseen point-forced results with the LM numerical data is very good, there are some shortcomings in using this modification. For small time-scale (high-frequency) motion the unsteady Oseen force (2.2) reduces to the Basset history force. Since the unsteady Oseen force scales linearly with the magnitude of 

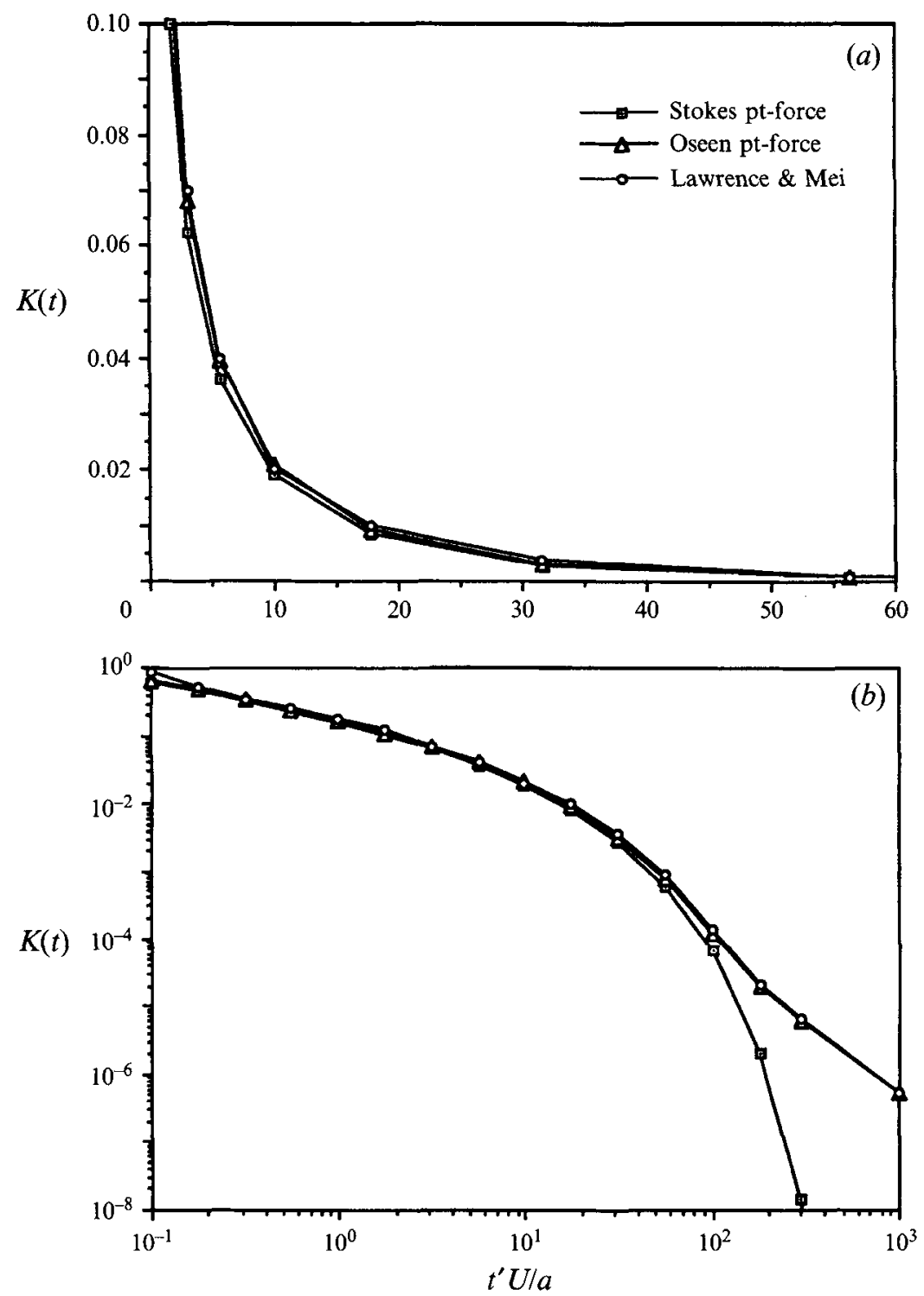

FiguRE 2. Comparison of the history force obtained from the present solution (2.5) with that from the previous result of Lovalenti \& Brady (2.4) and the numerical results of Lawrence \& Mei for a sphere making a step change in its speed at $t^{\prime}=0$ from $R e_{d}=0.1$ to $R e_{d}=0.3$ where $R e_{d}$ is the Reynolds number based on sphere diameter: $(a)$ linear plot; $(b)$ log plot.

the chosen point force, the modified expression for small time will reduce to a 'Basset' force that is altered by a factor of $1+O(R e)$ due to the $O(R e)$-corrected point force. This introduces an error in the hydrodynamic force of $O\left(a R e /(\tau v)^{1 / 2}\right)$ for small times, where $\tau$ is the characteristic time scale for changes in the motion of the body (or, for the case of an impulsive change in motion, it is equivalent to the time since the change occurred). This error would become significant for small time scales of order the diffusive time scale $a^{2} / v$ or smaller. Therefore, in order to maintain $O(R e)$ accuracy in the hydrodynamic force, this modification of the unsteady Oseen force 
should be employed for dynamic calculations when the time scale of variation for the motion satisfies $\tau \gg a^{2} / v$. As a final note, the modification changes the steady state value of the unsteady Oseen force (2.7) at $O\left(R e^{2}\right)$, but leaves unaltered its value at $O(R e)$.

\section{Long-time behaviour of the force on a body of arbitrary shape}

Next we turn our attention to the behaviour of the unsteady Oseen force acting on a nonspherical body. In the LB paper it was shown that the unsteady Oseen force acting on a body (of arbitrary shape) that impulsively accelerates from rest at time zero and assumes a steady rectilinear velocity thereafter is given by

$$
\begin{aligned}
\boldsymbol{F}_{U O S}(t)=\boldsymbol{F}_{\text {Sano }}(t) \equiv & \frac{3}{8} \operatorname{Re} \Phi \cdot\left[\left\{\left(1+\frac{4}{t^{2}}\right) \operatorname{erf}\left(\frac{t^{1 / 2}}{2}\right)+\frac{2}{(\pi t)^{1 / 2}}\left(1-\frac{2}{t}\right) \exp \left(-\frac{t}{4}\right)\right\} \boldsymbol{F}_{S t}^{\|}\right. \\
& \left.+\left\{\left(\frac{3}{2}-\frac{2}{t^{2}}\right) \operatorname{erf}\left(\frac{t^{1 / 2}}{2}\right)+\frac{3}{(\pi t)^{1 / 2}}\left(1+\frac{2}{3 t}\right) \exp \left(-\frac{t}{4}\right)\right\} \boldsymbol{F}_{S t}^{\perp}\right]
\end{aligned}
$$

where the subscript 'Sano' refers to the researcher who first derived the expression for a sphere (Sano 1981). Equation (3.1) is derived from (2.2) by performing the time integration. Note that for the rectilinear motion of a sphere $\boldsymbol{F}_{S t}^{\perp}=0$ and the second term in curly brackets in (3.1) is eliminated. Note also that in all cases of rectilinear motion the $\perp$ superscript designates a lift force perpendicular to the direction of motion.

Now when the body has been moving before the impulsive acceleration to its final steady velocity, another contribution must be added to (3.1) - the part of the history integral of (2.2) from $t=-\infty$ to 0 :

$$
\begin{aligned}
\boldsymbol{F}_{\text {UOs }}(t)= & \boldsymbol{F}_{\text {Sano }}(t)-\frac{3}{8} \operatorname{Re} \Phi \cdot\left\{\int _ { - \infty } ^ { 0 } \left[\left\{\frac{1}{|\boldsymbol{A}|^{2}}\left(\frac{\pi^{1 / 2}}{2|\boldsymbol{A}|} \operatorname{erf}(|\boldsymbol{A}|)-\exp \left(-|\boldsymbol{A}|^{2}\right)\right)\right\} \boldsymbol{F}_{S t}^{\|}(s)\right.\right. \\
& \left.\times\left\{\exp \left(-|\boldsymbol{A}|^{2}\right)-\frac{1}{2|\boldsymbol{A}|^{2}}\left(\frac{\pi^{1 / 2}}{2|\boldsymbol{A}|} \operatorname{erf}(|\boldsymbol{A}|)-\exp \left(-|\boldsymbol{A}|^{2}\right)\right)\right\} \boldsymbol{F}_{S t}^{1}(s)\right] \\
& \left.\times \frac{2 \mathrm{~d} s}{\pi^{1 / 2}(t-s)^{3 / 2}}\right\} .
\end{aligned}
$$

If the initial motion of the body is at a steady velocity in the same direction but different speed from the final motion, then (3.2) yields an exponential decay. This result implies that the history integral in (3.2) behaves as the negative of $\boldsymbol{F}_{\text {Sano }}(t)$ for large time. Since both contributions scale linearly with the Stokes drag at their respective speeds, the long-time behaviour of the history integral in (3.2) must be

$$
-\frac{3}{8} \operatorname{Re} \Phi \cdot\left\{\frac{\left(\boldsymbol{F}_{S t}^{\|}\right)_{i}}{b} \frac{4}{t^{2}}-\frac{\left(\boldsymbol{F}_{S t}^{\perp}\right)_{i}}{b} \frac{2}{t^{2}}\right\},
$$

where $b$ is the ratio of the initial speed to the final speed, and the subscript $i$ indicates the initial speed. Now if the Stokes drag quantities in (3.1) and (3.2) are replaced by their Oseen-corrected counterparts, there will no longer be exponential decay because of the nonlinearity of the Oseen relationship, and the long-time behaviour will be 

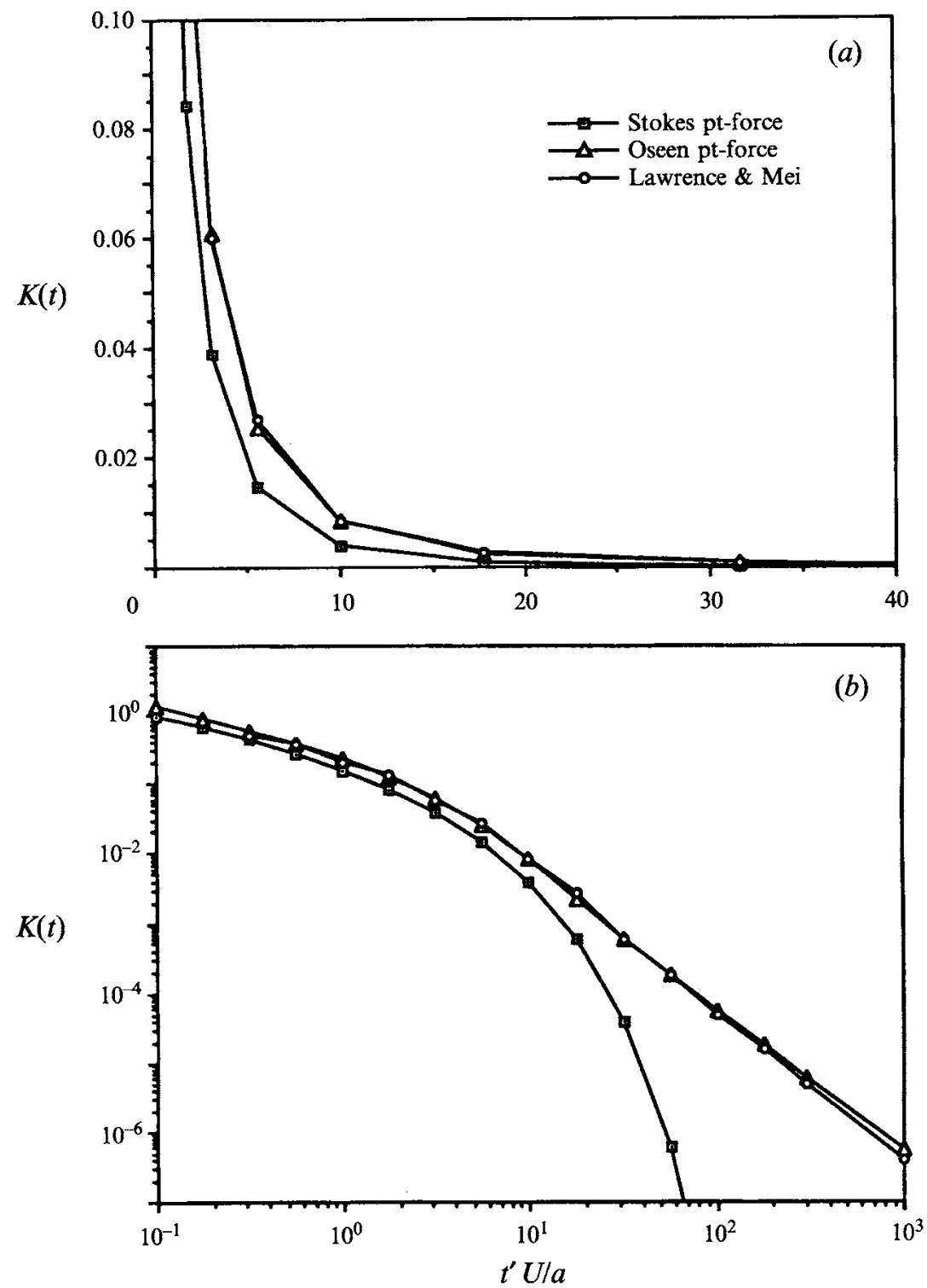

FIGURE 3. Comparison of the history force obtained from the present solution (2.5) with that from the previous result of Lovalenti \& Brady (2.4) and the numerical results of Lawrence \& Mei for a sphere making a step change in its speed at $t^{\prime}=0$ from $R e_{d}=0.8$ to $R e_{d}=1.0$ where $R e_{d}$ is the Reynolds number based on sphere diameter: $(a)$ linear plot; $(b)$ log plot.

given by

$$
\begin{aligned}
\boldsymbol{F}_{U O S}^{\prime}(t) \sim \frac{3}{8} \operatorname{Re} \Phi & \cdot\left\{\left(\boldsymbol{F}_{O s}^{\|}\right)_{f}+\frac{3}{2}\left(\boldsymbol{F}_{O s}^{\perp}\right)_{f}\right. \\
+ & \left.\left(\left(\boldsymbol{F}_{O s}^{\Downarrow}\right)_{f}-\frac{\left(\boldsymbol{F}_{O s}^{\|}\right)_{i}}{b}\right) \frac{4}{t^{2}}-\left(\left(\boldsymbol{F}_{O_{S}}^{\perp}\right)_{f}-\frac{\left(\boldsymbol{F}_{O s}^{\perp}\right)_{i}}{b}\right) \frac{2}{t^{2}}\right\}
\end{aligned}
$$


where the subscripts $i$ and $f$ indicate the initial and final values respectively. Note that the characteristic speed $U$ is chosen as the final speed. We note also that this expression differs from LM by the addition of the last decaying term which depends on $F_{O S}^{\perp}$ and, in general, may also contribute to the drag. Substituting for the Oseen force from (2.6), (3.4) can be simplified to

$$
\begin{aligned}
\boldsymbol{F}_{U O_{S}}^{\prime}(t) \sim & \frac{3}{8} \operatorname{Re} \Phi \cdot\left\{\left(\boldsymbol{F}_{O s}^{\|}\right)_{f}+\frac{3}{2}\left(\boldsymbol{F}_{O s}^{\perp}\right)_{f}\right. \\
& +\frac{3}{8} \operatorname{Re} \frac{(1-b)}{t^{2}}\left[9(\Phi \cdot \boldsymbol{p}) \cdot(\Phi \cdot \boldsymbol{p}) \boldsymbol{p}-3(\Phi: \boldsymbol{p} \boldsymbol{p})^{2} \boldsymbol{p}\right. \\
& -3 \Phi \cdot \Phi \cdot \boldsymbol{p}+(\Phi: \boldsymbol{p} \boldsymbol{p}) \Phi \cdot \boldsymbol{p}]\},
\end{aligned}
$$

where $\boldsymbol{p}$ is a unit vector in the direction of motion of the body.

\section{Force response to nonrectilinear motion}

All of the previous discussions have focused on the force response to changes in speed without a change in the direction of motion. The analytical expression (2.2) for the unsteady Oseen force is applicable to arbitrary motion, including curvilinear motion. Therefore, in order to investigate the force response to a nonrectilinear change in motion, we evaluated the unsteady Oseen-force for the case of a sphere making an abrupt right-angle change in its velocity direction. For the condition where the speed of the sphere was unchanged, the force response exhibited exponential decay for both components in the direction of original and final velocity, independent of whether the 'Oseen' modification (the procedure used above of replacing the Stokes quantities in (2.2) with their Oseen-corrected counterparts) was employed. $\dagger$ If there was a corresponding change in speed along with the right-angle change in velocity, the unsteady force again exhibited exponential decay; however, with the 'Oseen' modification, the decay was $t^{-2}$ for the component of the force in the direction of final velocity and exponential for the component in the direction of original velocity. The coefficient of this $t^{-2}$ decay agreed with the theoretical prediction made by LM, that this coefficient is determined solely by the change in speed of the body, which is independent of its change in direction of motion. Indeed, we found the coefficient to be the same as in (3.5), with $\Phi=I$ (the idem tensor) and $p$ in the direction of the final velocity. Figure 4 shows the modified history force (2.5) in the direction of final velocity for the case of a sphere making a right-angle change in its motion for various values of $b$, the ratio of initial to final speed. While initially the behaviour of the force is nearly identical for each case (as the unsteady Stokes solution would predict), the long-time behaviour is very different. For the case of $b=0$ (a sphere accelerating from rest), the decay is $t^{-2}$; for $0<b<1$, the decay is also $t^{-2}$ after a period of exponential decay when the force magnitude drops from $O(R e)$ to $O\left(R e^{2}\right)$; for $b=1$ the decay is exponential; and for $1<b<\infty$ (a sphere decelerating after the turn) the force drops off rapidly and actually becomes negative (a negative force here represents a force on the sphere pushing it in its direction of motion) and finally assumes the $t^{-2}$ decay given by (3.5).

Next we investigated the influence of the change in the direction of motion at an

$\dagger$ This finding does not preclude the existence of other higher-order (in $R e$ ) effects that may decay slower and thereby overtake this exponential decay, since, strictly speaking, the accuracy in the force expression $(2.1)$ is only to $O(R e)$. 


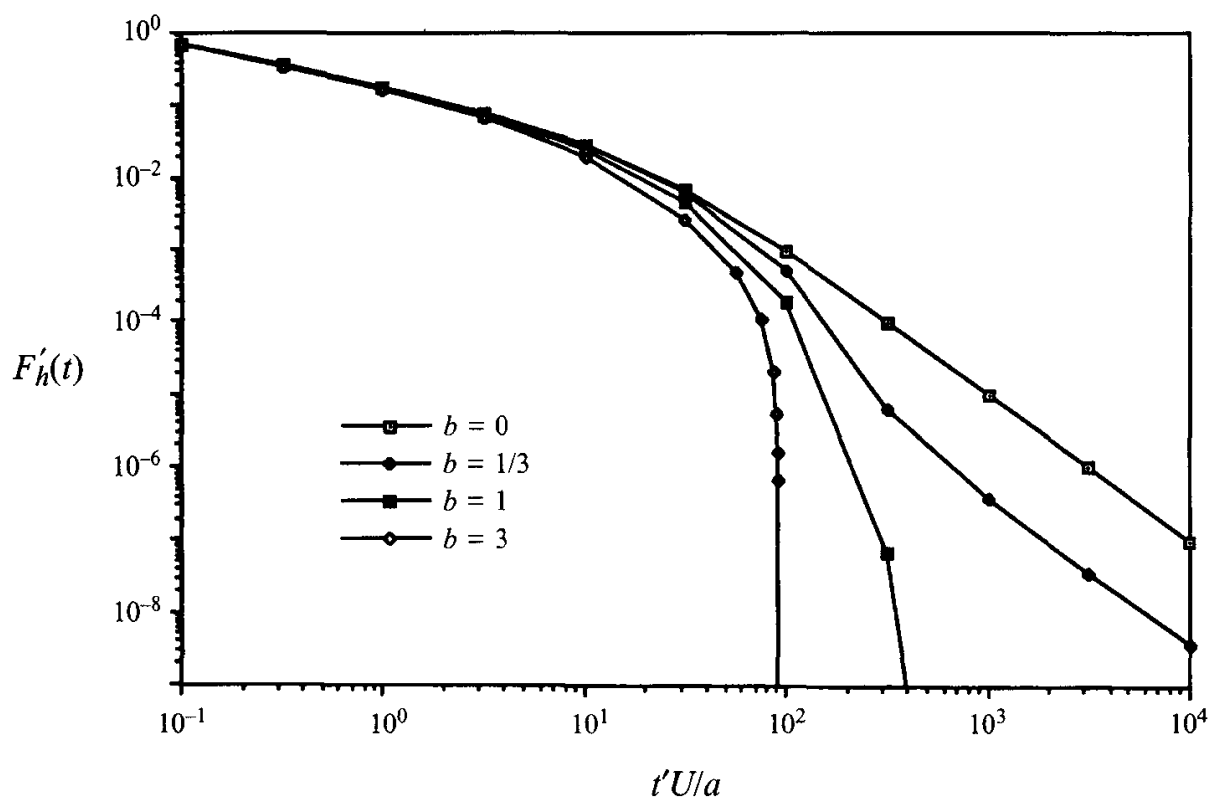

Figure 4. The response of the modified history force (2.5) on a sphere to a right-angle change in its motion at $t^{\prime}=0$ for various values of $b$, where $b$ is the ratio of the initial to the final speed. The Reynolds number (based on the sphere diameter and its final speed) is 0.3 .

arbitrary angle on the behaviour of the transient force. Figure 5 shows the modified history force (2.5) on a sphere in response to an abrupt change in velocity for various values of $\theta$, where $\theta$ is the angle between the initial and final rectilinear trajectories. The case of $\theta=0$ corresponds to a sphere making an exact reversal in its direction of motion; $\theta=\pi$ is for no change in direction, only a change in speed. It is interesting to consider the case of a body making a near reversal in its motion along with a change in speed. Initially the transient part of the force would decay as the Basset history force, as $t^{-1 / 2}$. Then, as the body made its way through its old wake (produced by its initial motion before the change in direction) the force would decay as $t^{-1}$. As the body emerged from the boundary of its old wake the decay would become exponential (at least for small Reynolds number) as the transient force drops from $O(R e)$ to $O\left(R e^{2}\right)$. This should occur for a time satisfying

$$
t^{\prime} \frac{U}{a}>\frac{1}{R e} \frac{1+\cos \theta / b}{\sin ^{2} \theta} \text {. }
$$

Finally the force would assume the $O\left(R e^{2}\right) t^{-2}$ decay described by (3.5). This behaviour is exhibited in figure 5 for the case of $\theta=0.2$. In all cases of $\theta>0$ the ultimate decay will be as (3.5) predicts.

\section{Conclusions}

At this point it is advantageous to discuss the appropriate hydrodynamic force expression to use under various conditions for bodies in motion in a time-dependent uniform flow. If the time scale for the variation of the motion of the body, $\tau$, satisfies $\tau \ll v / U^{2}$ the unsteady Stokes solution for the hydrodynamic force on the body (which neglects fluid convective effects) represent a valid approximation for the force, 


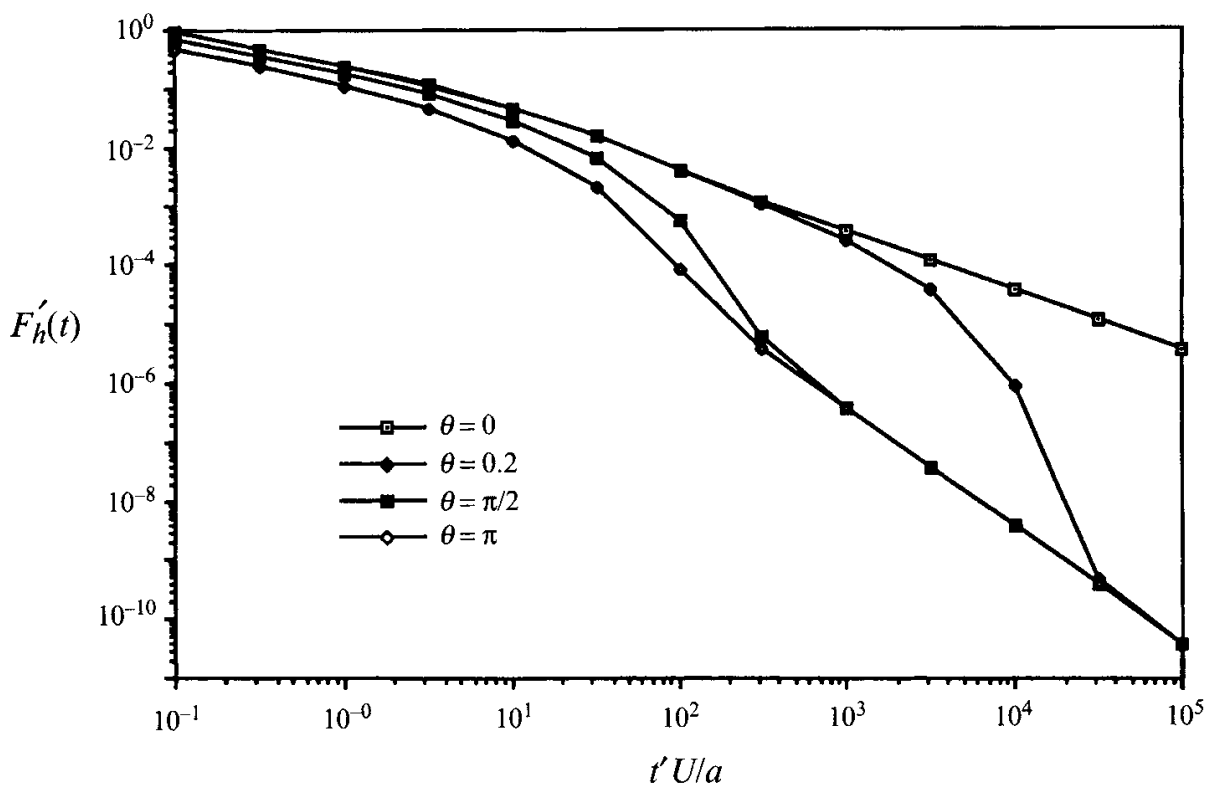

FIGURE 5. The response of the modified history force (2.5) on a sphere to an abrupt change in its velocity at $t^{\prime}=0$ for various values of $\theta$, where $\theta$ is the angle between the initial and final rectilinear trajectories. The Reynolds number (based on the sphere diameter and its final speed) is 0.3 and the ratio of the initial to the final speed is $1 / 3$.

independent of the magnitude of the Reynolds number. In this case, for a sphere, (2.1) can be used with the unsteady Oseen force replaced by the simpler Basset force. As the time scale for the motion of the body becomes $O\left(v / U^{2}\right)$ or greater, convective effects become important and need to be taken into account. For $O(R e)$-accurate trajectory calculations of spheres in arbitrary motion at small but finite Reynolds number, the expression given by (2.1) is appropriate since it incorporates these convective effects to leading order. If one is more interested in the long-time-scale behaviour (or long-time tail) of the force, however, (2.1) can again be employed with the use of the modified unsteady Oseen force (2.7), which appears to be valid for magnitudes of the Reynolds number of $O(1)$ or smaller. This modification can actually be reliably applied with $O(R e)$ accuracy for arbitrary motion of bodies, including bubbles and drops, provided the time scale is much greater than $a^{2} / v$. This modification also allows the force expression to capture all the long-time decays described in $\$ \$ 3$ and 4.

For Reynolds numbers greater than $O(1)$ no analytical dynamic force expressions exist. Although several semi-empirical expressions have been proposed, e.g. Odar \& Hamilton (1964) and Mei \& Adrian (1992), none can singlehandedly take into account the wide variety of transient behaviour that the hydrodynamic force can possess over the entire spectrum of time scales. The difficulty is rooted in the fact that the force response at finite Reynolds number is, in general, highly nonlinear with respect to the velocity of the body and has a strong dependence on the change in the direction of motion as well as the speed of the body. If one wishes to empirically (or semi-empirically) construct a dynamic force expression, the history force should be defined to behave as the history force from the unsteady Stokes solution for small time and, for large time, possess the steady convective contributions to the hydrodynamic force including the appropriate temporal decays. By using known solutions for the 
steady drag it is possible to alter the unsteady Oseen force so that it has the proper long-time finite Reynolds number temporal decays. However, the accuracy of the expression in representing the unsteady Stokes solution for small time would suffer severely at large Reynolds number, at best providing qualitative agreement.

As a final point of interest, consider the following definition of the history force as used in this paper and by others (Mei \& Adrian 1992): the part of the total hydrodynamic force left over after one removes contributions from the steady-state solution for the drag (this ensures the history force decays to zero at steady state), the added mass, and the acceleration of the imposed flow. With this definition, the history force at finite Reynolds number must possess fluid convective effects $\dagger$ for short time scales to negate the convective effects in the steady-state drag, since the total hydrodynamic force should not possess convective effects in the small time limit. For the case of a body making an abrupt change in motion, this history force at short times would behave similarly (that is, have the same temporal decay) for various Reynolds numbers, but would have a constant offset which depends on the Reynolds number. This property can give rise to inaccurate empirical expressions (Odar \& Hamilton 1964) and the seemingly anomalous (although correct) results that the history force (as defined above) depends on convective effects for small times (Mai, Klausner \& Lawrence 1994) which would lead one to believe erroneously that convective effects are present in the total hydrodynamic force for small times and that the unsteady Stokes solution for the total force at finite $R e$ is inaccurate for small times. In contrast, the unsteady Oseen force does not fulfil the above definition of the history forceł because it does not decay to zero at steady state. However, the unsteady Oseen force also does not possess fluid convective effects for small time scales, or at least these effects decrease as the time scale decreases. In addition, it properly possesses steady convective effects for large time scales when the transient behaviour of the total hydrodynamic force and fluid convective effects are intimately connected.

\section{REFERENCES}

LAWRENCE, C. J. \& MEI, R. 1995 Long-time behaviour of the drag on a body in impulsive motion. J. Fluid Mech. 283, 307-327 (referred to herein as LM).

Lovalenti, P. M. \& BRADY, J. F. 1993 The hydrodynamic force on a rigid particle undergoing arbitrary time-dependent motion at small Reynolds number. J. Fluid Mech. 256, 561-605 (referred to herein as LB).

MEI, R. \& AdRIAN, R. J. 1992 Flow past a sphere with an oscillation in the free-stream velocity and unsteady drag at finite Reynolds number. J. Fluid Mech. 237, 323-341.

Mei, R., Klausner, J. F. \& Lawrence, C. J. 1994 A note on the history force on a spherical bubble at finite Reynolds number. Phys. Fluids 6, 418-420.

OdAR, F. \& HAmilton, W. S. 1964 Force on a sphere accelerating in a viscous fluid. J. Fluid Mech. 18, 302-314.

SANo, T. 1981 Unsteady flow past a sphere at low Reynolds number. J. Fluid Mech. 112, 433-441.

$\dagger$ The convective effects referred to here are those that vary with the Reynolds number when time is scaled diffusively by $a^{2} / v$. The convective effects (or Reynolds number dependence) should decay away from the total hydrodynamic force as the time scale decreases.

$\ddagger$ Note that the unsteady Oseen force is itself a 'history force' since it is an integral over all the past history of the body's motion. Note also that because the unsteady Oseen force is nonlinear in the body's velocity, an integration kernel cannot be extracted that depends solely on time. It is this distinction from the Basset history integral that allows the unsteady Oseen force to exhibit the wide variety of temporal decays shown in this paper. 